Anaesthesist 2018 $\cdot 67: 1-2$

https://doi.org/10.1007/s00101-017-0403-4

(c) Springer Medizin Verlag GmbH, ein Teil von Springer Nature 2018

CrossMark

\title{
M. Zoller
}

Klinik für Anästhesiologie der LMU, Klinikum Großhadern, München, Deutschland

\section{„Antibiotic stewardship landauf landab"}

nen und Ärzte für eine rationale Antibiotikatherapie [5].

Insbesondere Anästhesisten und Intensivmediziner sind hier gefordert sich fortzubilden, weil wir unseren Patienten sowohl in der Intensivmedizin als auch in der Anästhesie täglich Antibiotika verordnen bzw. verabreichen. Antibiotische Therapien können nicht nur Resistenzen fördern, die Verabreichung eines Antibiotikums über mehrere Tage setzt z. B. auch das Mikrobiom eines Patienten einem selektiven Druck aus, der die intestinale Mikrobiota für ein Jahr verändern kann $[6,7]$. Es wird höchste Zeit, uns der Verantwortung einer rationalen Antibiotikatherapie zu stellen, im Sinne des einzelnen Patienten zur optimalen Verwendung von Antibiotika [8], aber auch im Sinne der Nachhaltigkeit zur Optimierung von Ressourcennutzung [9] und der Vermeidung von Resistenzentwicklungen.

\section{Hilfestellung oder Einschränkung?}

plan der WHO zu Antibiotikaresistenzen. [3]

Auch auf dem G20(Gruppe der Zwanzig)-Gipfel in diesem Jahr in Hamburg wurden die Bekämpfung antimikrobieller Resistenzen und eine bessere Kontrolle des Antibiotikaeinsatzes bei Menschen in der Abschlusserklärung nochmals bekräftigt; zudem wurde die Erarbeitung nationaler Aktionspläne gefordert [4]. Der vom Bundeskabinett schon 2 Jahre zuvor verabschiedete Nationale Aktionsplan „DART 2020 - AntibiotikaResistenzen bekämpfen zum Wohl von Mensch und Tier" sieht im "antibiotic stewardship" (ABS) eine besondere Bedeutung für die Fortbildung der Ärztin-
Der Beitrag von Lanckohr und Bracht ist ein wichtiger Schritt in diese Richtung. Der Beitrag beschreibt sehr gut die grundsätzlichen Strategien, die Evidenz und Möglichkeiten der Anwendungen mit Perspektiven im Alltag. Dies führt hoffentlich zu einem vermehrten Interesse an ABS-Fortbildungen und deren Anwendung. Ärztekammern und Fachgesellschaften sind hier zudem gefragt, ausreichende und geeignete Weiterbildungen flächendeckend anzubieten, um den zukünftigen Bedarf an Fort- und Weiterbildungen zu decken.

Allen Vorteilen zum Trotz kann ABS letztendlich aber auch als Einschränkung der ärztlichen Therapiefreiheit gesehen werden, v. a. bei den Intensivmedizinern, die grundsätzlich eine weitreichende Kompetenz auf dem Gebiet der Infektiologie haben. Vorstellbar ist auch, dass es gerade in der Akutmedizin bei Einzelfällen $\mathrm{zu}$ einer Verzögerung der Antibiotikagabe kommen könnte [10], außerdem könnten ABS-Teams durchaus auch für Sparmaßnahmen der kaufmännischen Direktion von Kliniken eingesetzt und dann potenziell missbraucht werden. Insbesondere unbeabsichtigte Folgen restriktiver Eingriffe des ABS in die antibiotische Therapie behandelter Ärztinnen und Ärzte sind bisher noch nicht gut untersucht [10]. Angesichts der Tatsache, dass die Mehrzahl der Untersuchungen zum ABS in angloamerikanischen Ländern durchgeführt wurde und dort häufig - besonders auf Intensivstationen - eine andere Delegations- und Entscheidungskultur besteht, wäre es angezeigt, in der deutschen Krankenhauslandschaft die Auswirkungen der Maßnahme auf das PatientenOutcome, den Antibiotikaverbrauch sowie den Wissenszuwachs in der Breite der Anwender mit wissenschaftlichen Methoden zu überprüfen.

In vielen Fällen sind zwar die von nationalen Institutionen entwickelten, klinischen Leitlinien angepasst an die Krankenhauslandschaft und umfassend, aber ihre Empfehlungen können möglicherweise schwer auf einzelne Patienten oder Kliniken angewendet werden. Die eigentlich anspruchsvolle Aufgabe für das ABS und die dazugehörigen Ärztinnen und Ärzte in den Kliniken liegt daher darin, darauf zu achten, dass mit diesen Maßnahmenbündeln des ABS kein Nachteil für den einzelnen Patien- 
ten entsteht, sondern die Kompetenzen in der rationalen Antibiotikatherapie verbessert und die Herausforderungen in der Zukunft gemeistert werden.

\section{Zoller}

\section{Korrespondenzadresse}

\section{Dr. M. Zoller}

Klinik für Anästhesiologie der LMU, Klinikum Großhadern

Marchioninistraße 15, 81377 München, Deutschland

Michael.zoller@med.uni-muenchen.de

Interessenkonflikt. M.Zoller gibt an, dass kein Interessenkonflikt besteht.

\section{Literatur}

1. WHO (2011) Strategischer Aktionsplan zur Bekämpfung von Antibiotikaresistenzen der WHO von 2011. http://www.euro.who.int/_ data/assets/pdf file/0010/147736/wd14G AntibioticResistance_111382bhn.pdf. Zugegriffen:01.01.2018

2. With K, Allerberger F, Amann Set al (2013) S3-Leitlinie "Strategien zur Sicherung rationaler Antibiotika-Anwendung im Krankenhaus". http://www. awmf.org/uploads/tx_szleitlinien/092-001I_ S3_Antibiotika_Anwendung_im_Krankenhaus_ 2013-verlaengert.pdf.Zugegriffen:01.01.2018

3. G7 Staaten oder Presse- und Informationsamt der Bundesregierung (2015) Abschlusserklärung G7-Gipfel. https://www.bundesregierung.de/ Content/DE/_Anlagen/G7_G20/2015-06-08-g7abschluss-deu.pdf? _blob $=$ publicationFile $\& v=5$. Zugegriffen: 01.01.2018

4. Presse-und Informationsamt derBundesregierung (2017) Erklärung der Staats- und Regierungschefs G20 Gipfel 2017. https://www.g20germany. de/Content/DE/_Anlagen/G7_G20/G20Abschlusserklaerung.pdf;jsessionid=7A49301 BF54DED0E76813824C4B6DF12.s2t1? blob= publicationFile\&v=7.Zugegriffen:01.01.2018

5. Bundesregierung. DART 2020 - Antibiotika-Resistenzen bekämpfen zum Wohl von Mensch und Tier https://www.bundesgesundheitsministerium.de/ fileadmin/Dateien/Publikationen/Ministerium/ Broschueren/BMG_DART_2020_Bericht_dt.pdf. Zugegriffen:01.01.2018

6. Rashid MU, Zaura E, Buijs MJ et al (2015) Determining the long-term effect of antibiotic administration on the human normal intestinal microbiota using culture and pyrosequencing methods. Clin Infect Dis 60(Suppl 2):S77-S84

7. Modi SR, Collins JJ, Relman DA (2014) Antibiotics and the gut microbiota. J Clin Invest 124(10):4212-4218

8. Paget J, Lescure D, Versporten A, et al (2017) European Commission. EU Guidelines for the prudent use of antimicrobials in human health. https://ec.europa.eu/health/amr/sites/amr/files/ amr_arna_report_20170717_en.pdf. Zugegriffen:01.01.2018

9. Barlam TF, Cosgrove SE, Abbo LM et al (2016) Implementing an antibiotic stewardship program: guidelines by the Infectious Diseases Society of
America and the Society for Healthcare Epidemiology of America. Clin Infect Dis 15;62(10):e51-77

10. Davey P, MarwickCA, Scott CLet al (2017) Interventions to improve antibiotic prescribing practices for hospital inpatients. Cochrane Database Syst Rev 9;2:CD3543
Forschungsgruppe des European Resuscitation Council (ERC) erhält lan G. Jacobs Award der American Heart Association (AHA) für internationale Forschungskooperation

Der lan G. Jacobs Award ehrt internationale Forschungskollaborationen im Bereich der erweiterten Reanimationsforschung. Auf dem Resuscitation Science Symposium der American Heart Association (AHA) ist die europäische Forschungsgruppe des European Resuscitation Council (ERC) um Prof. Dr. Bernd Böttiger, Uniklinik Köln, ausgezeichnet worden. Die Gruppe erhielt die Auszeichnung für ihre Forschungsaktivitäten im Rahmen des Europäischen Reanimationsregisters.

Die Wissenschaftler erhoben In der bisher größten europäischen Registerstudie „EuReCa ONE“ im Jahr 2014 erstmals auBerklinische Reanimationsergebnisse aus 27 europäischen Ländern gleichzeitig, um sie hinsichtlich Inzidenz, Versorgungsprozessen und Ergebnissen bzw. Outcome zu vergleichen. Das europäische Register wurde im Jahr 2007 unter dem Dach des ERC mit Unterstützung von Prof. Dr. Bernd Böttiger, Uniklinik Köln, als damaligem Präsident, gegründet.

Der lan G. Jacobs Award wird seit 2010 an internationale Forschungsgruppen verliehen, die sich in besonderer Weise im Bereich der erweiterten Reanimationsversorgung engagieren und hierbei richtungsweisende Ergebnisse mit hoher wissenschaftlicher Relevanz für die Versorgung von Menschen mit Herzkreislaufstillstand hervorbringen. Der Award wird von der AHA zu Ehren von Professor lan George Jacobs, PhD, OStJ, FERC, FAHA verliehen, der sich unermüdlich dafür eingesetzt hat, Mediziner und Wissenschaftler unterschiedlichster Disziplinen weltweit zusammen zu bringen, um die globale Versorgung von Menschen mit plötzlichem Herzkreislaufstillstand zu verbessern.

Mehr Informationen unter

https://www.eureca-one.eu

Quelle: Uniklinik Köln, www.uk-koeln.de 